\title{
Finite-Time Stabilization Control for a Rigid Spacecraft under Parameter Uncertainties
}

\author{
Aihua Zhang, Jianfei Ni, and Xing Huo \\ College of Engineering, Bohai University, Jinzhou 121000, China \\ Correspondence should be addressed to Aihua Zhang; jsxinxi_zah@163.com
}

Received 26 June 2014; Revised 11 July 2014; Accepted 15 July 2014; Published 4 August 2014

Academic Editor: Changzhu Zhang

Copyright (C) 2014 Aihua Zhang et al. This is an open access article distributed under the Creative Commons Attribution License, which permits unrestricted use, distribution, and reproduction in any medium, provided the original work is properly cited.

A novel finite-time control scheme is investigated for a rigid spacecraft in present of parameter uncertainties and external disturbances. Firstly, the spacecraft mathematical model is transformed into a cascading system by introducing an adaptive variable. Then a novel finite-time attitude stabilization control scheme for a rigid spacecraft is proposed based on the homogeneous method. Lyapunov stability analysis shows that the resulting closed-loop attitude system is proven to be stable in finite time without parameter uncertainties and asymptotically stable with parameter uncertainties. Finally, numerical simulation examples are also presented to demonstrate that the control strategy developed is feasible and effective for spacecraft attitude stabilization mission.

\section{Introduction}

With the development of aerospace science and technology faster and faster, for its higher technical requirements also, the spacecraft's attitude control and stability got close attention and extensive research. In recent years, many scholars in this field at home and abroad have made a lot of research results, such as optimal control [1], feedback control [2], input-output approach [3], adaptive control [4], T-S fuzzy theory [5-7], sliding mode control [8,9], data driven [10], and all this integrated control methods [11, 12]. These nonlinear control methods have certain advantages and are widely used in spacecraft control, although they can guarantee the stability of the closed-loop control system, and only for the equilibrium point, and the stable time is infinite. From the view of time optimal control system, the finitetime control is the optimal control scheme. Moreover, the finite-time controller has the fractional power which makes itself has the better robust performance than the normal controller, thus the cause in spacecraft attitude control system is widely researched $[13,14]$. In the existing research results, most of the control methods can only guarantee system converge to equilibrium point when the party time tends to infinity, and the finite time control method, to some extent has fast response, high control precision, and interference suppression.

For the closed-loop system research and prove the stability of the finite time in a row, primarily based on two criteria. The first method, mainly is to analysis based on the Lyapunov stability theorem, including terminal sliding mode and its deformation such as nonsingular terminal sliding mode, fast terminal sliding mode, and double exponential fast terminal sliding mode, mainly applied to general theorem of finite time and limited time extension theorem, and so forth. Another method based on the theory of homogeneous method is validated. In [13], a six-degree-of-freedom relative motion model is built aiming for the dynamic cooperative formation control $\mathrm{p}$ of master-slave spacecraft; then a terminal slide mode controller is proposed which guaranteed that the dynamic cooperative formation error can be in desired track. In [15] for using unit quaternion describing rigid spacecraft attitude tracking problem in the system, two kinds of finite time controller were designed to ensure the attitude of the attitude control system to expect accessibility of limited time, but the singular value phenomenon exists in the controller design. according to the standard terminal sliding mode control of singular value problem, the literature [16] has carried on the improvement on the basis of this, putting 
forward a kind of novel nonsingular terminal sliding mode control scheme, implementing the finite time stable attitude tracking control system.

Method based on the theory of homogeneous finite time stability studies, in less space in the field of application, is a new research hotspot to cause the attention of many scholars and attention. For only the position measurement information of space robot arm control system, the literature [17] proposed a class of output feedback combined with expected gravity compensation and continuous PD control, to solve the global problem of finite time control, and the system stability can be proved via Lyapunov theorem and homogeneous finite-time theorem. In addition, the literature [18] according to rigid satellite attitude control problem, design the existence and there is no outside interference torque under the condition of two kinds of finite time state feedback control law, Which in view of the situation without disturbances, based on the nature of nonlinear homogeneous systems, designs a kind of PD control feedback control algorithm, the position closed-loop control system of finite time convergences to equilibrium, and the method has simple structure and is convenient for application in the engineering practice. Aiming at the finite-time attitude stabilization issue of rigid, [19] gives a further control scheme. In this finite-time controller, a local continuous saturation finite-time controller and a global finite-time controller are designed, the two controller can be switched via a switch function, then the attitude control system could realized stability in finite-time. However, it regrets that the controller doesn't consider the effect about external disturbance.

In view of the presence of parameter uncertainty spacecraft attitude stability control system, it puts forward the category of odd theory based on the finite time control. First of all, by introducing new adaptive state variables, the spacecraft attitude control system is converted to a cascade system; Then a novel finite-time attitude stabilization control scheme for a rigid spacecraft is proposed based on the homogeneous method. Lyapunov stability analysis show that the resulting closed-loop attitude system is proven to be stable in finite time without parameter uncertainties, and asymptotically stable with parameter uncertainties, and there is no finite time stability of closed-loop system parameter uncertainties. Finally, the proposed algorithm is applied to a rigid spacecraft attitude stability control task; the digital simulation results verify the validity and feasibility of the scheme. And design in this paper, finite time controller structure is simple, is easy to adjust, and has the very high practical engineering practical value.

\section{The System Model and the Preliminary Knowledge}

2.1. The Spacecraft Attitude Dynamics and Kinematics Equation. Method to describe the kinematics of spacecraft attitude has direction cosine, Euler angle, quaternion, Rodrigo parameters, and modified Rodrigo (MRPs). Because the parameters of the quaternion description with minimal said aircraft motion attitude and global no singularity, so in this paper, quaternion is used to describe the spacecraft attitude kinematics:

$$
\dot{\mathbf{q}}=\frac{1}{2} \boldsymbol{\Xi}(\mathbf{q}) \boldsymbol{\omega}=\frac{1}{2}\left[\begin{array}{c}
-\mathbf{q}_{v}^{T} \\
q_{0} \mathbf{I}_{3}+\mathbf{q}_{v}^{\times}
\end{array}\right] \boldsymbol{\omega} .
$$

Type $\mathbf{q}=\left[q_{0} ; \mathbf{q}_{v}^{T}\right] \in \mathbf{R} \times \mathbf{R}^{3}$ expresses this system relative to the center of the earth of the inertial system unit quaternion and satisfaction $\mathbf{q}_{v}=\left[q_{1} ; q_{2} ; q_{3}\right] ; q_{0}^{2}+\mathbf{q}_{v}^{T} \mathbf{q}_{v}=1$ is the revision of this system relative to the inertial system of Rodrigo parameters; $\mathbf{I}_{3}$ is the $3 \mathrm{~d}$ unit matrix; $\boldsymbol{\omega}=\left[\omega_{1} ; \omega_{2} ; \omega_{3}\right]$ express this system inertial coordinate system of the rotating angular velocity relative to the center of the component in this system. Definition $\mathbf{Q}\left(\mathbf{q}_{v}\right)=q_{0} \mathbf{I}_{3}+\mathbf{q}_{v}^{\times}$, by type (1) to be seen

$$
\dot{\mathbf{q}}_{v}=\frac{1}{2}\left(q_{0} \mathbf{I}_{3}+\mathbf{q}_{v}^{\times}\right) \boldsymbol{\omega}=\frac{1}{2} \mathbf{Q} \boldsymbol{\omega} .
$$

Here, the definition of cross-product $(\cdot)^{\times}$as a skew symmetric matrix, that is to say, for a three-dimensional vector $\boldsymbol{\sigma}=\left[\sigma_{1} ; \sigma_{2} ; \sigma_{3}\right], \boldsymbol{\sigma}^{\times}$is expressed as

$$
\boldsymbol{\sigma}^{\times}=\left[\begin{array}{ccc}
0 & -\sigma_{3} & \sigma_{2} \\
\sigma_{3} & 0 & -\sigma_{1} \\
-\sigma_{2} & \sigma_{1} & 0
\end{array}\right] .
$$

Considering the spacecraft attitude dynamics equation

$$
\mathrm{J} \dot{\boldsymbol{\omega}}+\boldsymbol{\omega}^{\times} \mathrm{J} \boldsymbol{\omega}=\mathbf{u}+\mathbf{d} .
$$

In the type, $\mathbf{J} \in \mathbf{R}^{3 \times 3}$ is positive definite symmetric matrix type, expressing the spacecraft's inertia matrix; $\mathbf{u}$ is the three axis attitude control torque for spacecraft; and $\mathbf{d}$ is the outside interference torque vector for spacecraft.

Hypothesis 1. The disturbing torque $\mathbf{d}$ is unknown but bounded and is a normal number $d_{0}$ of establishments $\|\mathbf{d}\|<$ $d_{0}, d_{0}$ is the upper bound of the unknown disturbance torque.

In fact, the moment of inertia of spacecraft is not known for sure, that is to say, between the moment of inertia and its nominal value of on-orbit spacecraft it has certain uncertainty bounded difference, that is, as a result, the spacecraft attitude dynamics equation can describe as follows:

$$
\mathrm{J}_{0} \dot{\omega}+\omega^{\times} \mathrm{J}_{0} \boldsymbol{\omega}=\mathbf{u}+\mathbf{f} .
$$

Among them, $\mathbf{f}=\mathbf{d}-\Delta \mathbf{J} \dot{\boldsymbol{\omega}}-\boldsymbol{\omega}^{\times}(\Delta \mathrm{J} \boldsymbol{\omega})$.

Hypothesis 2. The arbitrarily $t>0$, to exit $q_{0}(t) \neq 0$.

By definition $\mathbf{Q}\left(\mathbf{q}_{v}\right)=q_{0} \mathbf{I}_{3}+\mathbf{q}_{v}^{\times}$, the definition, $\operatorname{det}(\mathbf{Q})=$ $q_{0}$. Therefore, through this hypothesis, we know that matrix $\mathbf{Q}\left(\mathbf{q}_{v}\right)$ is singular, namely, the $\boldsymbol{\omega}=2 \mathbf{Q}^{-1}\left(\mathbf{q}_{v}\right) \dot{\mathbf{q}}_{v}$ is singular. Thus, the system equation can be modified via (1) and (5) as for

$$
\begin{aligned}
& \mathbf{M}\left(\mathbf{q}_{v}\right) \ddot{\mathbf{q}}_{v}+\mathbf{C}\left(\mathbf{q}_{v}, \dot{\mathbf{q}}_{v}\right) \dot{\mathbf{q}}_{v}=\frac{1}{2} \mathbf{F}^{T}\left(\mathbf{q}_{v}\right) \mathbf{u}+\frac{1}{2} \mathbf{F}^{T}\left(\mathbf{q}_{v}\right) \mathbf{f} . \\
& \text { Among them, } \mathbf{F}=\mathbf{Q}^{-1}, \mathbf{M}=\mathbf{F}^{T} \mathbf{J F}, \\
& \quad \mathbf{C}=-\mathbf{F}^{T}\left(\mathbf{J F} \dot{\mathbf{G}}(\boldsymbol{\sigma})+2(\mathbf{J F} \dot{\boldsymbol{\sigma}})^{\times}\right) \mathbf{F}, \\
& \quad \mathbf{f}=\mathbf{d}-2 \Delta \mathbf{J F}\left(\ddot{\mathbf{q}}_{v}-\dot{\mathbf{Q} F} \dot{\mathbf{q}}_{v}\right)-4\left(\mathbf{F} \dot{\mathbf{q}}_{v}\right)^{\times}\left(\Delta \mathbf{J F} \dot{\mathbf{q}}_{v}\right) .
\end{aligned}
$$


Hypothesis 3. The value of the uncertainty of moment of inertia of spacecraft $\Delta \mathbf{J}$ is unknown bounded, thus $\mathbf{f}$ is unknown but bounded, and there is a normal number $f_{0}$ that was established, $\|\mathbf{f}\|<f_{0}$.

The spacecraft's equivalent system of (6) has the following important properties.

Nature 1. Matrix $\mathbf{M}$ is a symmetrical positive definite matrix and $\dot{\mathbf{M}}-2 \mathbf{C}$ is a written symmetric matrix; namely, $\xi^{T}(\dot{\mathbf{M}}-$ 2C) $\boldsymbol{\xi}=0, \forall \boldsymbol{\xi} \in \mathbf{R}^{3 \times 1}$.

Nature 2. Matrix $\mathbf{M}\left(\mathbf{q}_{\mathbf{v}}\right), \mathbf{C}\left(\mathbf{q}_{\mathbf{v}}, \dot{\mathbf{q}}_{\mathbf{v}}\right)$ are bounded value, that's there is a positive constant $c_{M \text { max }}>c_{M \text { min }}>0$ and $c_{C \max }>$ $c_{C \text { min }}>0$, yes $0<c_{M \text { min }} \leq\left\|\mathbf{M}\left(\mathbf{q}_{\mathbf{v}}\right)\right\| \leq c_{M \text { max }}, 0<$ $c_{C \min }\left\|\dot{\mathbf{q}}_{\mathbf{v}}\right\| \leq\left\|\mathbf{C}\left(\mathbf{q}_{\mathbf{v}}, \dot{\mathbf{q}}_{\mathbf{v}}\right)\right\| \leq c_{C \max }\left\|\dot{\mathbf{q}}_{\mathbf{v}}\right\|$.

2.2. Definitions and Lemmas. To facilitate the limited time of attitude control system design, based on the nonlinear system stability and the stability of finite time, the following definitions and lemma are given.

Consider the following nonlinear autonomous system:

$$
\dot{\mathbf{x}}=\mathbf{f}(\mathbf{x}), \quad \mathbf{f}(0)=0, \quad \mathbf{x}(0)=\mathbf{x}_{0}, \quad \mathbf{x} \in \mathbf{R}^{n}
$$

f : $\mathbf{U}_{0} \rightarrow \mathbf{R}^{n}$ is the continuous function of the gather $\mathbf{U}_{0}$, then the system (8) has a unique solution for arbitrary initial value.

Definition 1. If the system (8) the balance $\mathbf{x}=0$, on the field $\mathbf{U} \subset \mathbf{U}_{0}$ type Lyapunov stability and finite time stable, it is locally finite time stable. Finite time stable, also is a function $T: U \backslash\{0\} \rightarrow(0, \infty)$, for $\forall \mathbf{x}_{0} \in \mathbf{U} \subset \mathbf{R}^{n}$, solution of system (8) is $\mathbf{s}_{f}\left(\mathbf{x}_{0}\right)=0$, and $t \in\left[0, T\left(\mathbf{x}_{0}\right)\right), \mathbf{s}_{f}\left(\mathbf{x}_{0}\right) \in U \backslash\{0\}$ when $t>T\left(\mathbf{x}_{0}\right), \lim _{t \rightarrow T\left(\mathbf{x}_{0}\right)} \mathbf{s}_{f}\left(\mathbf{x}_{0}\right)=0$. In particular, if $\mathbf{U}=\mathbf{R}^{n}$, then the system ( 8$)$ is globally finite time stable.

Definition 2 (see [20]). Consider the following nonlinear autonomous system:

$$
\dot{\mathbf{x}}=\mathbf{f}(\mathbf{x}), \quad \mathbf{f}(0)=0, \quad x \in \mathbf{R}^{n}
$$

where $\mathbf{f}(\mathbf{x})=\left[f_{1}(\mathbf{x}), f_{2}(\mathbf{x}), \ldots, f_{n}(\mathbf{x})\right]^{T}: \mathbf{R}^{n} \rightarrow \mathbf{R}^{n}$ as a vector function. If any $\epsilon>0$, there exist $\left(r_{1}, r_{2}, \ldots, r_{n}\right) \in \mathbf{R}^{n}$, among them $r_{i}>0(i=1,2, \ldots, n)$, making $\mathbf{f}(\mathbf{x})$ satisfied

$$
f_{i}\left(\epsilon^{r_{1}} x_{1}, \epsilon^{r 2} x_{2}, \ldots, \epsilon^{r n} x_{n}\right)=\epsilon^{r_{i}+k} f_{i}(\mathbf{x}) .
$$

Among them, $k \geq-\max \left\{r_{i}, i=1,2, \ldots, n\right\}$, and said $\mathbf{f}(\mathbf{x})$ is about $\left(r_{1}, r_{2}, \ldots, r_{n}\right)$ With the second degree $k$.

Lemma 3 (see [21]). Consider the following system:

$$
\dot{\mathbf{x}}=\mathbf{f}(\mathbf{x})+\widehat{\mathbf{f}}(\mathbf{x}), \quad \mathbf{f}(\mathbf{0})=\mathbf{0}, \quad \mathbf{x} \in \mathbf{R}^{n}
$$

where $\mathbf{f}(\mathbf{x})$ is a continuous homogeneous vector function, about $\left(r_{1}, r_{2}, \ldots, r_{n}\right) \in \mathbf{R}^{n}$ with homogeneous degree $k<0$, and meets $\widehat{\mathbf{f}}(\mathbf{0})=\mathbf{0}$. Hypothesis $\mathbf{x}=\mathbf{0}$ is a asymptotic stability of equilibrium point in the system $\dot{\mathbf{x}}=\mathbf{f}(\mathbf{x})$, if

$$
\begin{array}{r}
\lim _{\epsilon \rightarrow 0} \frac{f_{i}\left(\epsilon^{r_{1}} x_{1}, \epsilon^{r 2} x_{2}, \ldots, \epsilon^{r m} x_{m}\right)}{\epsilon^{r_{i}+k}}=0, \\
i=1,2, \ldots, n, \quad \forall \mathbf{x} \neq 0
\end{array}
$$

was established, then $\mathbf{x}=\mathbf{0}$ is a system (11) of a locally finite time stable equilibrium point.

In addition, if the system (11) is the global asymptotic stability and is locally finite time stable, then the system is globally finite time stable.

Lemma 4 ((Barbalat lemma) [22]). Set $\phi: \mathbf{R} \rightarrow \mathbf{R}$ is $[0, \infty)$, uniformly continuous function, assuming $\lim _{t \rightarrow \infty} \int_{0}^{t} \phi(\tau) d \tau$ that exists and is limited, $\lim _{t \rightarrow \infty} \phi(\tau)=0$ was established.

Lemma 5 (see [23]). Suppose $f(t): \mathbf{R}^{+} \rightarrow \mathbf{R}$ is a differentiable function and meets the following:

(1) $\lim _{t \rightarrow \infty} f(t)$ exists and is limited;

(2) there are two functions $g_{1}(t)$ and $g_{2}(t)$ satisfaction $\dot{f}(t)=g_{1}(t)+g_{2}(t)$;

if $g_{1}(t)$ is uniformly continuous function and meets $\lim _{t \rightarrow \infty} g_{2}(t)=0$, then, $\lim _{t \rightarrow \infty} g_{1}(t)=0$ is established.

\section{Design of the Control System}

To facilitate the design and analysis of the control system, we define vector $\operatorname{Tanh}(\boldsymbol{\epsilon}), \operatorname{sig}(\boldsymbol{\epsilon})^{\alpha} \in \mathbf{R}^{n}$, diagonal matrix $\operatorname{Sech}(\boldsymbol{\epsilon}) \in \mathbf{R}^{n \times n}$ is as follows:

$$
\begin{aligned}
\operatorname{Tanh}(\boldsymbol{\epsilon}) & =\left[\tanh \left(\epsilon_{1}\right), \ldots, \tanh \left(\epsilon_{n}\right)\right]^{T}, \\
\operatorname{sig}(\boldsymbol{\epsilon})^{\alpha} & =\operatorname{sign}(\epsilon)|\epsilon|^{\alpha} \\
& =\left[\operatorname{sign}\left(\epsilon_{1}\right)\left|\epsilon_{1}\right|^{\alpha}, \ldots, \operatorname{sign}\left(\epsilon_{n}\right)\left|\epsilon_{n}\right|^{\alpha}\right]^{T}, \\
\operatorname{Sech}(\boldsymbol{\epsilon}) & =\operatorname{diag}\left(\operatorname{sech}\left(\epsilon_{1}\right), \ldots, \operatorname{sech}\left(\epsilon_{n}\right)\right) .
\end{aligned}
$$

Type, $\boldsymbol{\epsilon}=\left[\epsilon_{1}, \ldots, \epsilon_{n}\right]^{T} \in \mathbf{R}^{n}, 0<\alpha<1, \tanh (\cdot)$ and $\operatorname{sech}(\cdot)$ said the standard hyperbolic tangent and hyperbolic secant function, respectively, $\operatorname{sign}(\cdot)$ said the standard of symbol function, $\operatorname{diag}(\cdot)$ said a standard nonzero diagonal matrix. According to the above definition, we can easily get the following conclusion:

$$
\begin{gathered}
\operatorname{Tanh}(\boldsymbol{\epsilon})^{T} \operatorname{sig}(\boldsymbol{\epsilon})^{\alpha} \geq \operatorname{Tanh}(\boldsymbol{\epsilon})^{T} \operatorname{Tanh}(\boldsymbol{\epsilon}) \\
\left.\epsilon_{i}\right|^{\alpha+1} \geq \tanh ^{2}\left(\epsilon_{i}\right) \\
\lambda_{M}\left(\operatorname{Sech}^{2}(\boldsymbol{\epsilon})\right)=1
\end{gathered}
$$

Among them, $\lambda_{M}(\cdot)$ and $\lambda_{m}(\cdot)$ express the maximum and minimum eigenvalues of respective matrix. 
Here in order to facilitate the description and analysis of the attitude control system, we define $\mathbf{x}=\left[\mathbf{x}_{1}, \mathbf{x}_{2}, \mathbf{x}_{3}\right]^{T}, \mathbf{x}_{1}=$ $\mathbf{q}_{v}, \mathbf{x}_{1}=\mathbf{q}_{v}, \mathbf{x}_{3}=\mathbf{v}$, the available:

$$
\begin{aligned}
& \dot{\mathbf{x}}_{1}=\dot{\mathbf{q}}_{v}=\mathbf{x}_{2}, \\
& \dot{\mathbf{x}}_{2}=\ddot{\mathbf{q}}_{v}=-\mathbf{M}^{-1}\left(\mathbf{C} \dot{\mathbf{q}}_{v}-\frac{1}{2} \mathbf{F}^{T}(\mathbf{u}+\mathbf{f})\right), \\
& \dot{\mathbf{x}}_{3}=\dot{\mathbf{v}} .
\end{aligned}
$$

Step 1. First, we give the Lyapunov function as follows:

$$
\begin{aligned}
V_{1}= & \frac{1}{\alpha+1} \sum_{i=1}^{3} k_{1}\left|\mathbf{x}_{1 i}\right|^{\alpha+1}+\frac{1}{2} \mathbf{x}_{2}^{T} \mathbf{M} \mathbf{x}_{2} \\
& +\rho \operatorname{Tanh}\left(\mathbf{x}_{1}\right)^{T} \mathbf{M}\left(\mathbf{x}_{1}\right) \mathbf{x}_{2}+\frac{1}{2} \mathbf{x}_{3}^{T} \mathbf{K}_{v} \mathbf{B}^{-1} \mathbf{x}_{3} .
\end{aligned}
$$

Type, $k_{1}>0$ and $\rho>0$ stay on the design on the number of normal and $\mathbf{K}_{v}$ is diagonal positive definite matrix for constant, $k_{v i}>0$ is the diagonal element of $\mathbf{K}_{v}, k_{v i}>0$ is the positive definite matrix, and there are

$$
\begin{aligned}
& \frac{1}{2(\alpha+1)} \sum_{i=1}^{3} k_{1}\left|\mathbf{x}_{1 i}\right|^{\alpha+1}+\frac{1}{4} \mathbf{x}_{2}^{T} \mathbf{M} \mathbf{x}_{2} \\
& \quad+\rho \operatorname{Tanh}\left(\mathbf{x}_{1}\right)^{T} M\left(\mathbf{x}_{1}\right) \mathbf{x}_{2} \\
& =\frac{1}{4}\left(\mathbf{x}_{2}+2 \rho \operatorname{Tanh}\left(\mathbf{x}_{1}\right)\right)^{T} \mathbf{M}\left(\mathbf{x}_{2}+2 \rho \operatorname{Tanh}\left(\mathbf{x}_{1}\right)\right) \\
& \quad-\rho^{2} \operatorname{Tanh}\left(\mathbf{x}_{1}\right)^{T} \mathbf{M} \operatorname{Tanh}\left(\mathbf{x}_{1}\right) \\
& \quad+\frac{1}{2(\alpha+1)} \sum_{i=1}^{3} k_{1}\left|\mathbf{x}_{1 i}\right|^{\alpha+1} \\
& \geq \sum_{i=1}^{3}\left(\frac{k_{1}}{2(\alpha+1)}-\rho^{2} c_{M \max }\right) \tanh ^{2}\left(\mathbf{x}_{1 i}\right) .
\end{aligned}
$$

If you select the appropriate parameters $k_{1}>0, \rho>0$, $c_{M \max }>0$, and $0<\alpha<1$ meet

$$
\frac{k_{1}}{2(\alpha+1)}-\rho^{2} c_{M \max }>0 .
$$

Type (17) into type (16), for $\mathbf{x}=\left[\mathbf{x}_{1}, \mathbf{x}_{2}, \mathbf{x}_{3}\right]^{T} \neq 0$ one has

$$
\begin{aligned}
V_{1}= & \sum_{i=1}^{3}\left(\frac{k_{1}}{2(\alpha+1)}-\rho^{2} c_{M \max }\right) \tanh ^{2}\left(\mathbf{x}_{1 i}\right) \\
& +\frac{1}{4}\left(\mathbf{x}_{2}+2 \rho \operatorname{Tanh}\left(\mathbf{x}_{1}\right)\right)^{T} \mathbf{M}\left(\mathbf{x}_{2}+2 \rho \operatorname{Tanh}\left(\mathbf{x}_{1}\right)\right) \\
& +\frac{1}{2(\alpha+1)} \sum_{i=1}^{3} k_{1}\left|\mathbf{x}_{1 i}\right|^{\alpha+1} \\
& +\frac{1}{4} \mathbf{x}_{2}^{T} \mathbf{M} \mathbf{x}_{2}+\frac{1}{2} \mathbf{x}_{3}^{T} k_{v} \mathbf{B}^{-1} \mathbf{x}_{3} \\
> & 0
\end{aligned}
$$

Defined type (16) of the Lyapunov functions are

$$
\begin{aligned}
\dot{V}_{1}= & \frac{1}{\alpha+1} \sum_{i=1}^{3} k_{1}\left|\mathbf{x}_{1 i}\right|^{\alpha+1}+\frac{1}{2} \mathbf{x}_{2}^{T} \mathbf{M} \mathbf{x}_{2} \\
& +\rho \operatorname{Tanh}\left(\mathbf{x}_{1}\right)^{T} \mathbf{M} \mathbf{x}_{2}+\frac{1}{2} \mathbf{x}_{3}^{T} \mathbf{K}_{v} \mathbf{B}^{-1} \mathbf{x}_{3} \\
= & k_{1} \mathbf{x}_{2}^{T} \operatorname{sig}\left(\mathbf{x}_{1}\right)^{\alpha}+\frac{1}{2} \mathbf{x}_{2}^{T} \dot{\mathbf{M}} \mathbf{x}_{2} \\
& -\mathbf{x}_{2}^{T}\left(\mathbf{C} \mathbf{x}_{2}-\frac{1}{2} \mathbf{F}^{T}(\mathbf{u}+\mathbf{f})\right)+\rho\left(\operatorname{Sech}^{2}\left(\mathbf{x}_{1}\right) \mathbf{x}_{2}\right)^{T} \mathbf{M} \mathbf{x}_{2} \\
& +\rho \operatorname{Tanh}\left(\mathbf{x}_{1}\right)^{T} \dot{\mathbf{M}} \mathbf{x}_{2}-\rho \operatorname{Tanh}\left(\mathbf{x}_{1}\right)^{T} \\
& \times\left(\mathbf{C} \mathbf{x}_{2}-\frac{1}{2} \mathbf{F}^{T}(\mathbf{u}+\mathbf{f})\right)+\mathbf{x}_{3}^{T} \mathbf{K}_{v} \mathbf{B}^{-1} \dot{\mathbf{x}}_{3} .
\end{aligned}
$$

At this point, we give the following control scheme:

$$
\begin{aligned}
& \mathbf{u}=2 \mathbf{Q}^{\mathrm{T}}\left(-\mathbf{K}_{v} \mathbf{x}_{3}-k_{1} \operatorname{sig}\left(\mathbf{x}_{1}\right)^{\alpha}-k_{2} \operatorname{sig}\left(\mathbf{x}_{2}\right)^{\beta}-k_{3} \mathbf{x}_{2}\right), \\
& \dot{\mathbf{x}}_{3}=-\mathbf{A} \mathbf{x}_{3}+\mathbf{B} \mathbf{x}_{2} .
\end{aligned}
$$

On the type, constant $k_{1}, k_{2}>0,0<\alpha, \beta<1$, positive definite matrices $\mathbf{A}, \mathbf{B}$ are undetermined parameters. Type (21) with type (22) into type (20) are

$$
\begin{aligned}
\dot{V}_{1}= & k_{1} \mathbf{x}_{2}^{T} \operatorname{sig}\left(\mathbf{x}_{1}\right)^{\alpha}+\frac{1}{2} \mathbf{x}_{2}^{T} \dot{\mathbf{M}} \mathbf{x}_{2} \\
- & \mathbf{x}_{2}^{T}\left(\mathbf{C} \mathbf{x}_{2}-\frac{1}{2} \mathbf{F}^{T}\right. \\
& \times\left(2 \mathbf { Q } ^ { T } \left(-\mathbf{K}_{v} \mathbf{x}_{3}-k_{1} \operatorname{sig}\left(\mathbf{x}_{1}\right)^{\alpha}-k_{2} \operatorname{sig}\left(\mathbf{x}_{2}\right)^{\beta}\right.\right. \\
& \left.\left.\left.-k_{3} \mathbf{x}_{2}\right)+\mathbf{f}\right)\right) \\
+ & \rho\left(\operatorname{Sech}^{2}\left(\mathbf{x}_{1}\right) \mathbf{x}_{2}\right)^{T} \mathbf{M} \mathbf{x}_{2}+\rho \operatorname{Tanh}\left(\mathbf{x}_{1}\right)^{T} \dot{\mathbf{M}} \mathbf{x}_{2} \\
+ & \mathbf{x}_{3}^{T} \mathbf{K}_{v} \mathbf{B}^{-1}\left(-\mathbf{A} \mathbf{x}_{3}+\mathbf{B} \mathbf{x}_{2}\right)-\rho \operatorname{Tanh}\left(\mathbf{x}_{1}\right)^{T} \\
& \times\left(\mathbf{C x}_{2}-\frac{1}{2} \mathbf{F}^{T} \quad\right. \\
& \times\left(2 \mathbf { Q } ^ { T } \left(-\mathbf{K}_{v} \mathbf{x}_{3}-k_{1} \operatorname{sig}\left(\mathbf{x}_{1}\right)^{\alpha}\right.\right. \\
& \left.\left.\left.-k_{2} \operatorname{sig}\left(\mathbf{x}_{2}\right)^{\beta}-k_{3} \mathbf{x}_{2}\right)+\mathbf{f}\right)\right)
\end{aligned}
$$




$$
\begin{aligned}
= & -\mathbf{x}_{3}^{T} \mathbf{K}_{v} \mathbf{B}^{-1} \mathbf{A} \mathbf{x}_{3}-\rho \operatorname{Tanh}\left(\mathbf{x}_{1}\right)^{T} k_{1} \operatorname{sig}\left(\mathbf{x}_{1}\right)^{\alpha} \\
+ & \mathbf{x}_{2}^{T}\left(-k_{2} \operatorname{sig}\left(\mathbf{x}_{2}\right)^{\beta}+\frac{1}{2} \mathbf{F}^{T} \mathbf{f}\right)-k_{3} \mathbf{x}_{2}^{T} \mathbf{x}_{2} \\
+ & \rho\left(\left(\operatorname{Sech}^{2}\left(\mathbf{x}_{1}\right) \mathbf{x}_{2}\right)^{T} \mathbf{M} \mathbf{x}_{2}+\operatorname{Tanh}\left(\mathbf{x}_{1}\right)^{T} \mathbf{C} \mathbf{x}_{2}\right. \\
& +\operatorname{Tanh}\left(\mathbf{x}_{1}\right)^{T}\left(-k_{2} \operatorname{sig}\left(\mathbf{x}_{2}\right)^{\beta}+\frac{1}{2} \mathbf{F}^{T} \mathbf{f}\right) \\
& \left.-k_{3} \operatorname{Tanh}\left(\mathbf{x}_{1}\right)^{T} \mathbf{x}_{2}\right)-\rho \operatorname{Tanh}\left(\mathbf{x}_{1}\right)^{T} \mathbf{K}_{v} \mathbf{x}_{3} .
\end{aligned}
$$

By Hypotheses 1 and 3, as well as Hypothesis 2 known nature, $\|\mathbf{F}\|=\left\|\mathbf{Q}^{-1}\right\|$ and $\|\mathbf{F}\|=\left\|\mathbf{Q}^{-1}\right\|$ are bounded, the definition $\mathbf{f}=(1 / 2) \mathbf{F f}=\left[\bar{f}_{1}, \bar{f}_{2}, \bar{f}_{3}\right]^{T}$, then there is $\bar{f}_{0}$ met $\left|\bar{f}_{i}\right|<\bar{f}_{0}$, thus

$$
\begin{aligned}
- & k_{2} \operatorname{sig}\left(\mathbf{x}_{2}\right)^{\beta}+\frac{1}{2} \mathbf{F f} \\
= & -k_{2} \operatorname{sig}\left(\mathbf{x}_{2}\right)^{\beta}+\overline{\mathbf{f}} \\
= & -\left(k_{2}-\operatorname{diag}\left(\left|\bar{f}_{i}\right|\right) \operatorname{diag}^{-1}\left(\operatorname{sig}\left(\mathbf{x}_{2}\right)^{\beta}\right)\right) \operatorname{sig}\left(\mathbf{x}_{2}\right)^{\beta} .
\end{aligned}
$$

If you choose the appropriate parameters $k_{2}-\left(\left|\bar{f}_{i}\right| /\left|x_{2 i}\right|^{\beta}\right)$ $=\gamma \geq 0$ make $k_{2}-\operatorname{diag}\left(\left|\bar{f}_{i}\right|\right) \operatorname{diag}^{-1}\left(\operatorname{sig}\left(\mathbf{x}_{2}\right)^{\beta}\right)$ for the positive definite matrix, in which $\mathbf{x}_{2}$ is now under control within the boundary layer $\left|x_{2 i}\right|^{\beta} \leq\left|\bar{f}_{i}\right| / k_{2}$, there are

$$
\begin{aligned}
\mathbf{x}_{2}^{T} & \left(-k_{2} \operatorname{sig}\left(\mathbf{x}_{2}\right)^{\beta}+\frac{1}{2} \mathbf{F}^{T} \mathbf{f}\right) \\
& =-\mathbf{x}_{2}^{T}\left(k_{2}-\operatorname{diag}\left(\left|\bar{f}_{i}\right|\right) \operatorname{diag}^{-1}\left(\operatorname{sig}\left(\mathbf{x}_{2}\right)^{\beta}\right)\right) \operatorname{sig}\left(\mathbf{x}_{2}\right)^{\beta} \\
& =-\gamma\left\|\mathbf{x}_{2}\right\|^{\beta+1} \leq 0 .
\end{aligned}
$$

According to the nature of the assumptions and functional equation, then there is

$$
\begin{gathered}
\rho\left(\left(\operatorname{Sech}^{2}\left(\mathbf{x}_{1}\right) \mathbf{x}_{2}\right)^{T} \mathbf{M} \mathbf{x}_{2}+\operatorname{Tanh}\left(\mathbf{x}_{1}\right)^{T} \mathbf{C x}_{2}\right. \\
+\operatorname{Tanh}\left(\mathbf{x}_{1}\right)^{T}\left(-k_{2} \operatorname{sig}\left(\mathbf{x}_{2}\right)^{\beta}+\frac{1}{2} \mathbf{F}^{T} \mathbf{f}\right) \\
\left.\quad-k_{3} \operatorname{Tanh}\left(\mathbf{x}_{1}\right)^{T} \mathbf{x}_{2}\right) \\
\leq \rho\left(c_{M \max }+c_{C \max }+\gamma+k_{3}\right)\left\|\mathbf{x}_{2}^{2}\right\| .
\end{gathered}
$$

Here, $\delta \geq 0$ is the design for the stay constant.

$$
\begin{aligned}
\dot{V}_{1}= & -\mathbf{x}_{3}^{T} \mathbf{K}_{v} \mathbf{B}^{-1} \mathbf{A} \mathbf{x}_{3}-\rho \operatorname{Tanh}\left(\mathbf{x}_{1}\right)^{T} k_{1} \operatorname{sig}\left(\mathbf{x}_{1}\right)^{\alpha} \\
& +\mathbf{x}_{2}^{T}\left(-k_{2} \operatorname{sig}\left(\mathbf{x}_{2}\right)^{\beta}+\frac{1}{2} \mathbf{F}^{T} \mathbf{f}\right)-\rho \operatorname{Tanh}\left(\mathbf{x}_{1}\right)^{T} \mathbf{K}_{v} \mathbf{x}_{3} \\
& +\rho\left(\left(\operatorname{Sech}^{2}\left(\mathbf{x}_{1}\right) \mathbf{x}_{2}\right)^{T} \mathbf{M} \mathbf{x}_{2}+\operatorname{Tanh}\left(\mathbf{x}_{1}\right)^{T} \mathbf{C} \mathbf{x}_{2}\right. \\
& +\operatorname{Tanh}\left(\mathbf{x}_{1}\right)^{T}\left(-k_{2} \operatorname{sig}\left(\mathbf{x}_{2}\right)^{\beta}+\frac{1}{2} \mathbf{F}^{T} \mathbf{f}\right) \\
& \left.-k_{3} \operatorname{Tanh}\left(\mathbf{x}_{1}\right)^{T} \mathbf{x}_{2}\right)-k_{3} \mathbf{x}_{2}^{T} \mathbf{x}_{2} \\
\leq & -\mathbf{x}_{3}^{T}\left(\mathbf{K}_{v} \mathbf{B}^{-1} \mathbf{A}-\frac{\rho \lambda_{M}\left(\mathbf{K}_{v}\right)}{2 k_{1}}\right) \mathbf{x}_{3}-\gamma\left\|\mathbf{x}_{2}\right\|^{\beta+1} \\
& -\left(k_{3}-\rho\left(c_{M \max }+c_{C \max }+\gamma+k_{3}\right)\right)\left\|\mathbf{x}_{2}^{2}\right\| .
\end{aligned}
$$

If we select the appropriate parameters to satisfy the following conditions:

$$
\begin{gathered}
\left(\mathbf{K}_{v} \mathbf{B}^{-1} \mathbf{A}-\frac{\rho \lambda_{M}\left(\mathbf{K}_{v}\right)}{2 k_{1}}\right)>0, \\
\left(k_{3}-\rho\left(c_{M \text { max }}+c_{C \text { max }}+\gamma+k_{3}\right)\right)>0 .
\end{gathered}
$$

we can get $\dot{V}_{1} \leq 0$, and when $\mathbf{x}_{3}=\mathbf{x}_{2}=0$, meet $\mathbf{x}_{3}=\mathbf{x}_{2}=0$. With Lyapunov stability theorem and LaSalle lemma available spacecraft attitude control system (15) in the controller (21) and (22), select the appropriate parameters meeting the type (28), the system is asymptotically stable.

Step 2. In this section, we will prove that the system type (15) locally finite $\mathbf{f}=0$ is stable parameter uncertainty. First of all, we have to transform type (15):

$$
\begin{aligned}
& \dot{\mathbf{x}}_{1}=\mathbf{x}_{2}, \\
& \dot{\mathbf{x}}_{2}=-\mathbf{M}^{-1}\left(\mathbf{C} \dot{\mathbf{q}}-\frac{1}{2} \mathbf{F}^{T}(\mathbf{u}+\mathbf{f})\right), \\
& \dot{\mathbf{x}}_{3}=-\mathbf{A} \mathbf{x}_{3}+\mathbf{B} \mathbf{x}_{2}, \\
& \dot{\mathbf{x}}_{1}=\mathbf{x}_{2}, \\
& \dot{\mathbf{x}}_{2}=-\mathbf{M}^{-1}\left(\mathbf{C} \mathbf{x}_{2}-\frac{1}{2} \mathbf{F}^{T}\left(2 \mathbf { Q } ^ { T } \left(-\mathbf{K}_{v} \mathbf{x}_{3}-k_{1} \operatorname{sig}\left(\mathbf{x}_{1}\right)^{\alpha}\right.\right.\right. \\
& \dot{\mathbf{x}}_{3}=-\mathbf{A x}_{3}+\mathbf{B} \mathbf{x}_{2} .
\end{aligned}
$$


According to Lemma 3, we define $\widehat{f}_{2}=-\mathbf{M}^{-1}\left(\mathbf{C x}_{2}+\mathbf{K}_{v} \mathbf{x}_{3}+\right.$ $\left.(1 / 2) k_{2} \mathbf{F}^{T} \operatorname{sig}\left(\mathbf{x}_{2}\right)^{\beta}+(1 / 2) k_{3} \mathbf{F}^{T} \mathbf{x}_{2}-(1 / 2) \mathbf{F}^{T} \mathbf{f}\right), \hat{f}_{3}=-\mathbf{A} \mathbf{x}_{3}$, thus the type can be turned into

$$
\begin{aligned}
& \dot{\mathbf{x}}_{1}=\mathbf{x}_{2}, \\
& \dot{\mathbf{x}}_{2}=-k_{1} \mathbf{M}^{-1} \operatorname{sig}\left(\mathbf{x}_{1}\right)^{\alpha}+\widehat{f}_{2}, \\
& \dot{\mathbf{x}}_{3}=\mathbf{B} \mathbf{x}_{2}+\widehat{f}_{3} .
\end{aligned}
$$

Thus, we easily know the type of a standard system:

$$
\begin{aligned}
& \dot{\mathbf{x}}_{1}=\mathbf{x}_{2}, \\
& \dot{\mathbf{x}}_{2}=-k_{1} \mathbf{M}^{-1} \operatorname{sig}\left(\mathbf{x}_{1}\right)^{\alpha}, \\
& \dot{\mathbf{x}}_{3}=\mathbf{B} \mathbf{x}_{2} .
\end{aligned}
$$

The system is a homogeneous system $\left(r_{11}, r_{12}, \ldots, r_{1 n}\right.$, $\left.r_{21}, r_{22}, \ldots, r_{2 n}, r_{31}, r_{32}, \ldots, r_{3 n}\right) \in \mathbf{R}^{3 n}$ with $k=((\alpha-1) /(\alpha+$ 1)) $(0<\alpha<1)$ homogeneous degree of, including, $r_{1 i}=r_{1}=$ $2 /(\alpha+1), r_{2 i}=r_{2}=1, r_{3 i}=r_{3}=r_{1}$, according to Lemma 3 , we have

$$
\begin{aligned}
\lim _{\epsilon \rightarrow 0} & \frac{\widehat{f}_{2}}{\left(\epsilon^{r_{1}} \mathbf{x}_{1}, \epsilon^{r_{2}} \mathbf{x}_{2}, \epsilon^{r_{3}} \mathbf{x}_{3}\right)} \\
\epsilon^{r_{2}+k} & -\lim _{\epsilon \rightarrow 0}\left(\left(\mathbf{M}^{-1}\left(\epsilon^{r_{1}} \mathbf{x}_{1}\right)\left(\mathbf{C}\left(\epsilon^{r_{1}} \mathbf{x}_{1}, \epsilon^{r_{2}} \mathbf{x}_{2}\right)+\frac{1}{2} k_{3} \mathbf{F}^{T}\left(\epsilon^{r_{1}} \mathbf{x}_{1}\right)\right)\right.\right. \\
& \left.\left.\times \epsilon^{r_{2}} \mathbf{x}_{2}\right) \times\left(\epsilon^{r_{2}+k}\right)^{-1}\right) \\
& -\lim _{\epsilon \rightarrow 0} \frac{\mathbf{M}^{-1}\left(\epsilon^{r_{1}} \mathbf{x}_{1}\right) \mathbf{K}_{v} \epsilon^{r_{3}} \mathbf{x}_{3}}{\epsilon^{r_{2}+k}} \\
& -\frac{1}{2} \lim _{\epsilon \rightarrow 0} \frac{\mathbf{M}^{-1}\left(\epsilon^{r_{1}} \mathbf{x}_{1}\right) k_{2} \mathbf{F}^{T}\left(\epsilon^{r_{1}} \mathbf{x}_{1}\right) \operatorname{sig}^{\beta}\left(\epsilon^{r_{2}} \mathbf{x}_{2}\right)}{\epsilon^{r_{2}+k}} \\
& +\lim _{\epsilon \rightarrow 0} \frac{\mathbf{M}^{-1}\left(\epsilon^{r_{1}} \mathbf{x}_{1}\right) \mathbf{F}^{T}\left(\epsilon^{r_{1}} \mathbf{x}_{1}\right) \mathbf{f}}{\epsilon^{r_{2}+k}} \\
= & -\lim _{\epsilon \rightarrow 0} \mathbf{M}^{-1}(0)\left(\mathbf{C}\left(0_{1}, 0_{2}\right)+\frac{1}{2} k_{3} \mathbf{F}^{T}\left(0_{1}\right)\right) \epsilon^{-k} \mathbf{x}_{2} \\
& -\lim _{\epsilon \rightarrow 0} \mathbf{M}^{-1}(0) \mathbf{K}_{v} \epsilon^{r_{3}-r_{2}-k} \mathbf{x}_{3} \\
& -\frac{1}{2} \lim _{\epsilon \rightarrow 0} \mathbf{M}^{-1}(0) k_{2} \mathbf{F}^{T}\left(0_{1}\right) \operatorname{sig}^{\beta}\left(\mathbf{x}_{2}\right) \epsilon^{-k} \\
& +\lim _{\epsilon \rightarrow 0} \mathbf{M}^{-1}(0) \frac{\mathbf{F}^{T}\left(\epsilon^{r_{1}} \mathbf{x}_{1}\right) \mathbf{f}}{\epsilon^{r_{2}+k} .}
\end{aligned}
$$

If the parameter uncertainty $\mathbf{f}=0$, thus you can type to

$$
\begin{aligned}
& \lim _{\epsilon \rightarrow 0} \frac{\widehat{f}_{2}\left(\epsilon^{r_{1}} \mathbf{x}_{1}, \epsilon^{r_{2}} \mathbf{x}_{2}, \epsilon^{r_{3}} \mathbf{x}_{3}\right)}{\epsilon^{r_{2}+k}} \\
&=-\lim _{\epsilon \rightarrow 0} \mathbf{M}^{-1}(0)\left(\mathbf{C}\left(0_{1}, 0_{2}\right)+\frac{1}{2} k_{3} \mathbf{F}^{T}\left(0_{1}\right)\right) \epsilon^{-k} \mathbf{x}_{2}
\end{aligned}
$$
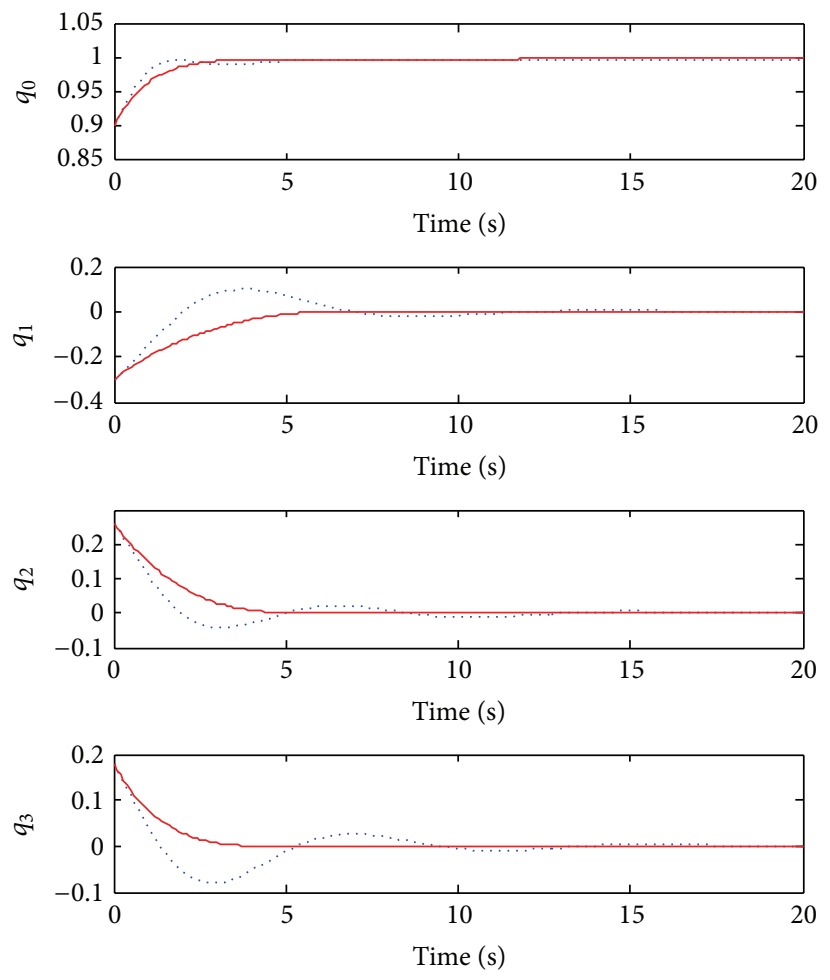

PID

FTC

FIgURE 1: Time responses of the attitude quaternion $\mathbf{q}$ without disturbs.

$$
\begin{aligned}
& -\lim _{\epsilon \rightarrow 0} \mathbf{M}^{-1}(0) \mathbf{K}_{v} \epsilon^{r_{3}-r_{2}-k} \mathbf{x}_{3} \\
& -\frac{1}{2} \lim _{\epsilon \rightarrow 0} \mathbf{M}^{-1}(0) k_{2} \mathbf{F}^{T}\left(0_{1}\right) \operatorname{sig}^{\beta}\left(\mathbf{x}_{2}\right) \epsilon^{-k}
\end{aligned}
$$$$
=0
$$

type, $r_{3}-r_{2}-k=2(1-\alpha) /(\alpha+1)>0$.

In the same way, we can get

$$
\begin{gathered}
\lim _{\epsilon \rightarrow 0} \frac{\widehat{f}_{3}\left(\epsilon^{r_{1}} \mathbf{x}_{1}, \epsilon^{r_{2}} \mathbf{x}_{2}, \epsilon^{r_{3}} \mathbf{x}_{3}\right)}{\epsilon^{r_{3}+k}} \\
=-\lim _{\epsilon \rightarrow 0} \frac{\mathbf{A} \epsilon^{r_{3}} \mathbf{x}_{3}}{\epsilon^{r_{3}+k}} \\
=-\lim _{\epsilon \rightarrow 0} \mathbf{A} \epsilon^{-k} \mathbf{x}_{3}=0 .
\end{gathered}
$$

Therefore, via Lemma 3, we can confirmed that the spacecraft attitude control system can realize finite-time local stabilization when $\mathbf{f}=0$ of parameters uncertainty. Thus, we give the following theorem.

Theorem 6. In view of the attitude control system (15), under the Assumptions 1-3, the finite time controllers (21) and (22), select the appropriate parameters meeting the type (28), the system is asymptotically stable. When parameter uncertainties $\mathbf{f}=0$, control system of spacecraft attitude can achieve partial finite time stable. 

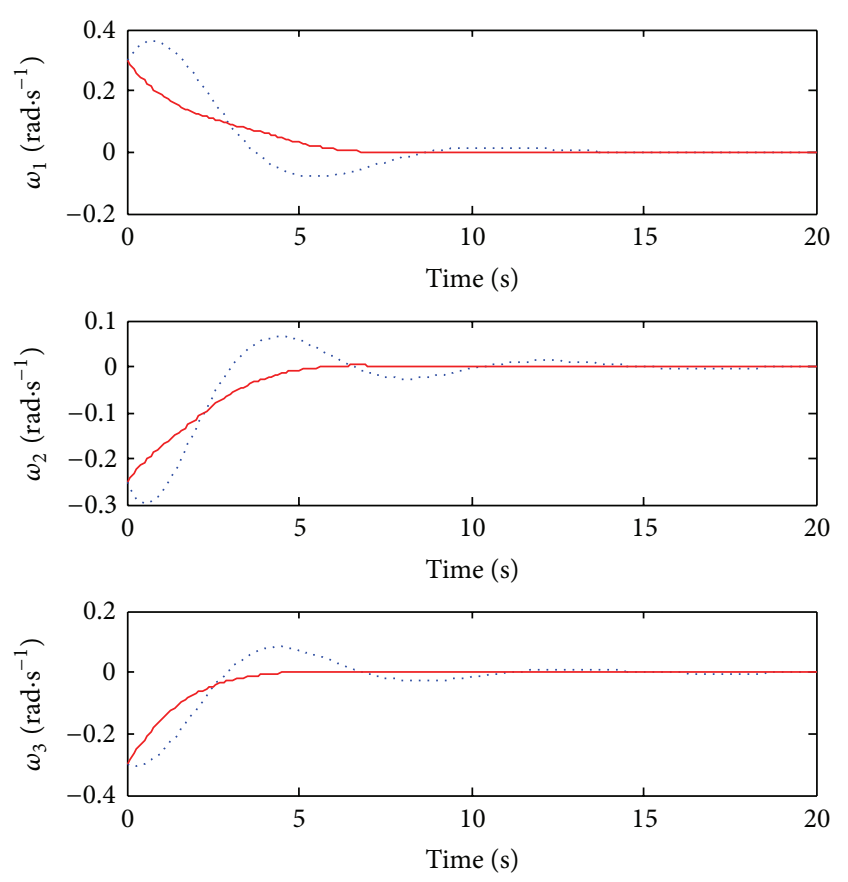

PID

— FTC

FIGURE 2: Time responses of the attitude angular velocity $\boldsymbol{\omega}$ without disturbs.
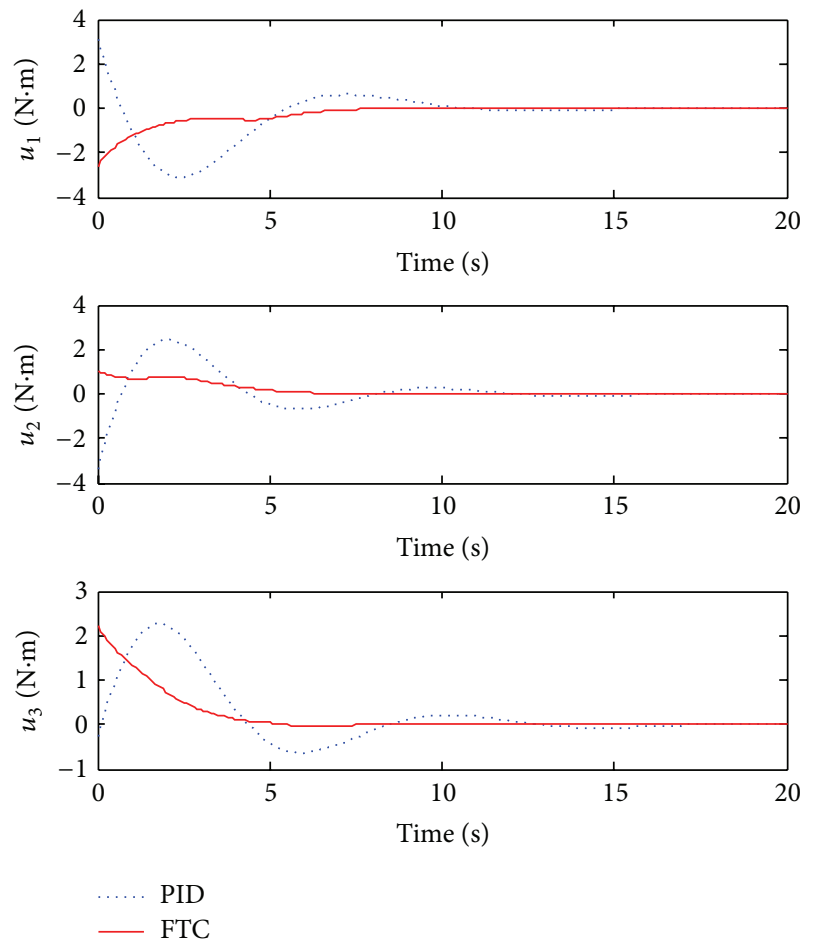

Figure 3: Time responses of the control torques $\mathbf{u}$ without disturbs.
Proof. See above Steps 1 and 2, just here are omitted.

\section{Simulation and Analysis}

In order to validate the proposed the validity of the finite time control (FTC), using MATLAB/Simulink software, for spacecraft attitude maneuver control process simulation, spacecraft main simulation parameters and controller parameters are shown in Table 1. Another hypothesis role in spacecraft external disturbance torque is

$$
\mathbf{d}(t)=10^{-2} *\left[\begin{array}{c}
3 \cos (10 a t)+4 \sin (3 a t) \\
-1.5 \sin (2 a t)+3 \cos (5 a t) \\
3 \sin (10 a t)-8 \sin (4 a t)
\end{array}\right] \mathrm{N} \cdot \mathrm{m}
$$

Type $a=0.8$.

Under the model parameters of the above, for the sake of comparison, this part for limited time controller designed in this paper (FTC) and the traditional classic PID control simulation is to verify the effectiveness of the scheme and superiority. In accordance with the process of the design of the controller, the simulation is divided into a spacecraft attitude control system without external disturbance and outside disturbance of two parts.

(A) Without External/Internal Disturbance. In situations where there is no disturbance torque, the finite time controller designed in this limited time (21) can make the control system to achieve stability. Figures 1 and 2 show the stance and attitude angular velocity of the spacecraft system time response curves. The spacecraft attitude control system can reach stabilization within 5 SEC under the proposed controller FTC (red solid line), and the stabilization precision can reach $10^{-4}$ also. The closed-loop control system has realized the finite-time stabilization. In addition the PID (blue line) under the action of the controller, the stable time of the system in more than $15 \mathrm{SEC}$, and stable precision only, even in the absence of interference, the attitude of the spacecraft has larger perturbation and steady-state error. Figure 3 shows that the controller without interference of provides the moment of time response curves. Thus, the traditional PID control can achieve asymptotic stability of control system, the FTC in this paper, the control can guarantee the stability of attitude control of the limited time and has the very good superiority.

(B) With External/Internal Disturbance. In view of the existence of disturbance torque, without changing the model and parameters, the controller of this paper, when compared with the traditional PID controller, is simulated. Figures 4 and 5 show the stance and attitude angular velocity of the spacecraft system time response curves. In the proposed controller FTC (red solid line), under the control of spacecraft attitude control system can achieve stability within 8 SEC, stable and has high level of accuracy, guarantee the stability of the closed-loop control system. Moreover, the effective inhibition ability to external disturbance of the proposed controller 

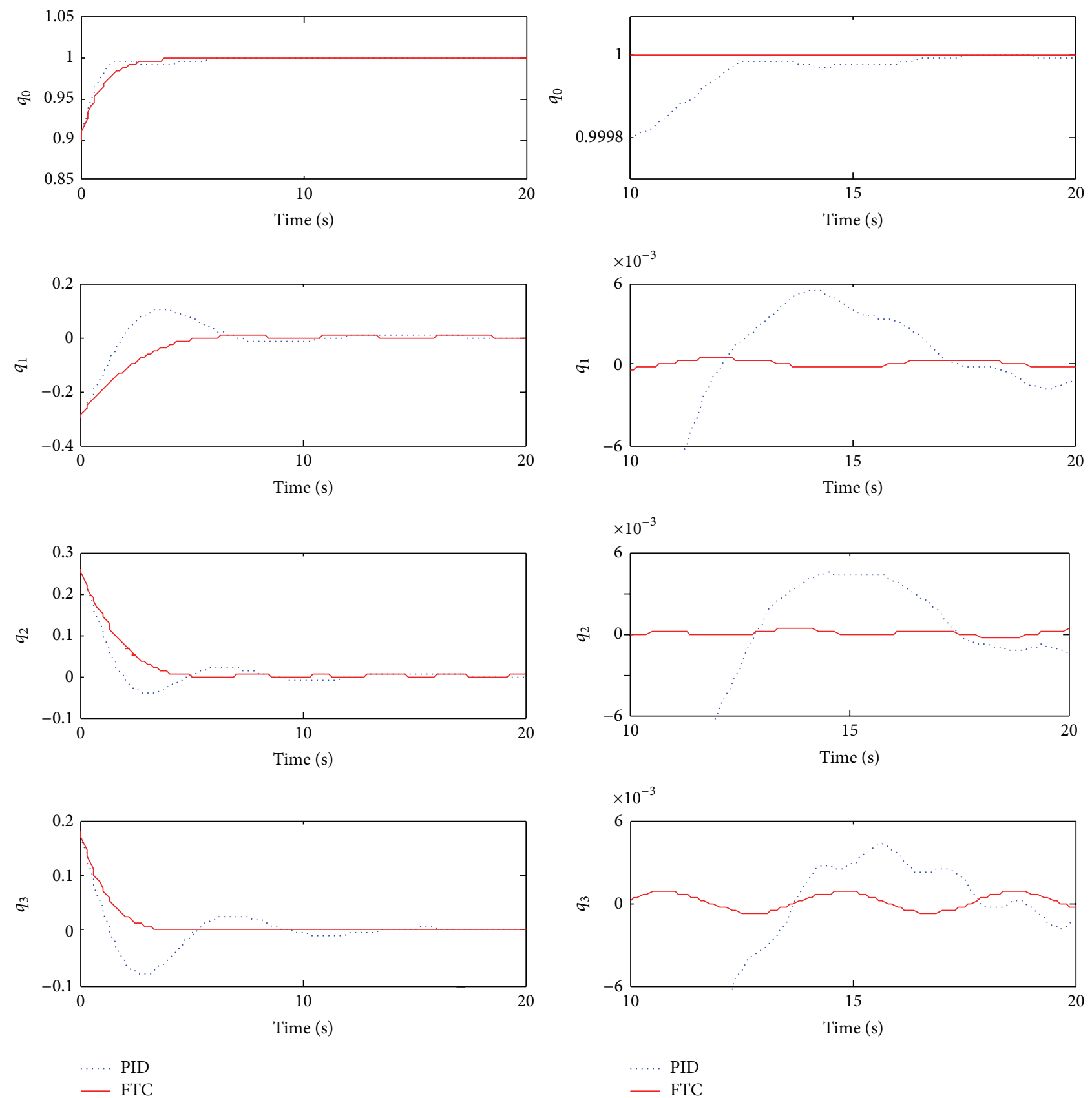

FIGURE 4: Time responses of the attitude quaternion $\mathbf{q}$ with disturbs.

TABLE 1: Spacecraft model and controller parameters.

\begin{tabular}{|c|c|}
\hline The project name & Parameter \\
\hline \multirow{3}{*}{ Spacecraft model parameters } & Moment of inertia $\mathbf{J}=\left[\begin{array}{lll}20 & 0 & 0.9 ;\end{array}\right]\left[\begin{array}{lll}0 & 17 & 0 ;\end{array}\right]\left[\begin{array}{lll}0.9 & 0 & 15\end{array}\right] \mathrm{kg} \cdot \mathrm{m}^{2}$ \\
\hline & Initial position $\mathbf{q}(0)=[0.9 ; \quad-0.3 ; 0.26 ; 0.18]$ \\
\hline & Initial angular velocity $\omega(0)=[0.3 ;-0.25 ;-0.3] \mathrm{rad} / \mathrm{s}$ \\
\hline \multirow{2}{*}{ Finite time controller } & $k_{1}=1.8, \quad k_{2}=1.2, \quad k_{3}=2.6, \quad \alpha=0.8, \quad \beta=0.86$ \\
\hline & $\mathbf{K}_{v}=\operatorname{diag}\left(\begin{array}{lll}1 & 1.2 & 2\end{array}\right)$ \\
\hline PID controller & $K_{I}=0.0005$ \\
\hline
\end{tabular}



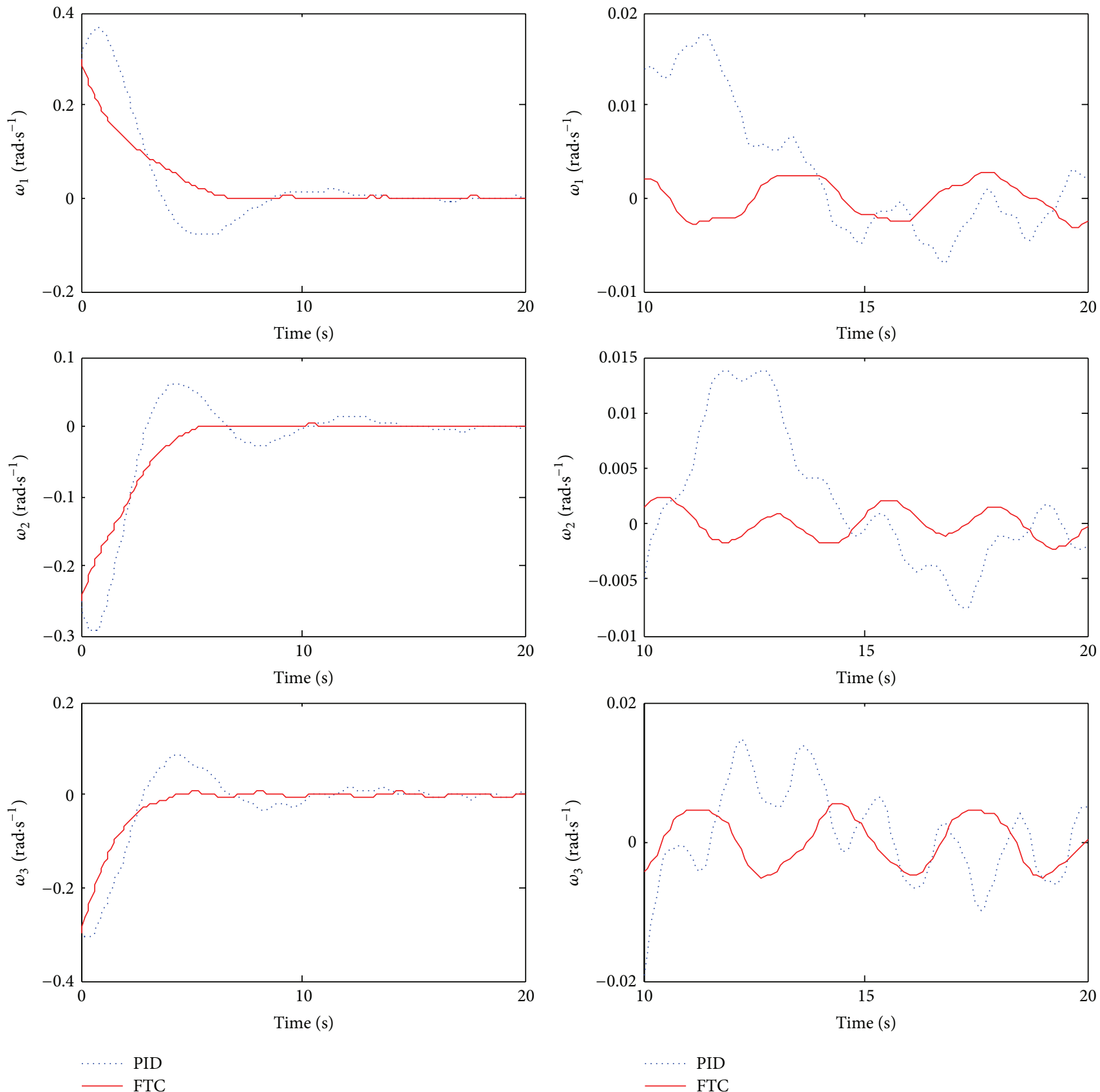

FIGURE 5: Time responses of the attitude angular velocity $\boldsymbol{\omega}$ with disturbs.

FTC can be seen in Figure 5, from the time responds curve of the spacecraft attitude can be found that the attitude almost have no chattering. In PID (blue line) under the action of the controller, the stable time of the system in more than 20 SEC, and stable precision only, under the action of external disturbance, the attitude of the spacecraft has larger perturbation and steady-state error. Figure 6 shows that the controllers in the absence of any disturbance provide the moment of time response curves. Thus, even in the presence of external disturbances, in this paper, the actual FTC control can guarantee the stability of attitude control of the limited time and has the very good superiority.

\section{Conclusions}

In view of the presence of parameter uncertainty spacecraft attitude stability control system, it puts forward the category of odd theory based on the finite time control. First of all, by introducing a new adaptive state variables, the spacecraft attitude control system is converted to a cascade system; 

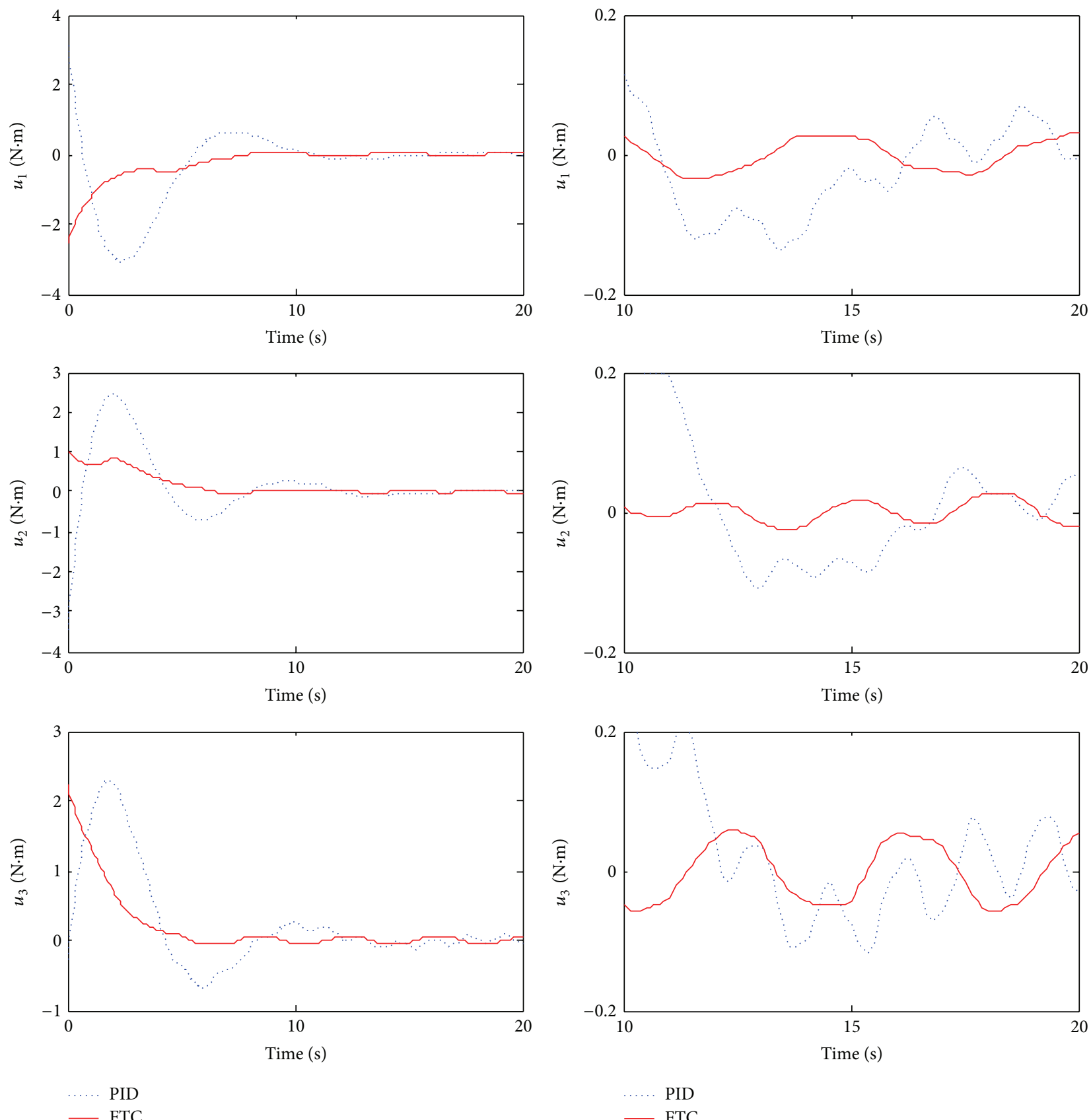

Figure 6: Time responses of the control torques $\mathbf{u}$ with disturbs.

then, based on the theory of homogeneity of the method, the finite time asymptotically stable controller design for a class of novel, and by using Lyapunov theory, proved that the system asymptotic stability in the presence of parameter uncertainties, and there is no finite time stability of closedloop system parameter uncertainties. Finally, the proposed algorithm is applied to a rigid spacecraft attitude stability control task; the digital simulation results verify the validity and feasibility of the scheme. And design in this paper, finite time controller structure is simple, easy to adjust, and has the very high practical engineering practical value. But, this paper studies to locally finite time stable, for spacecraft attitude control system of globally finite time stable controller design and argumentation, the difficulties and focus will be the next step of work.

\section{Conflict of Interests}

The authors declare that there is no conflict of interests regarding the publication of this paper. 


\section{Acknowledgments}

This present work was supported partially by National Natural Science Foundation of China (Project no. 61304149) and Natural Science Foundation of Liaoning, China (Project no. 2013020044). The authors highly appreciate the above financial supports.

\section{References}

[1] S. Yin, H. Luo, and S. Ding, Real-Time Implementation of FaultTolerant Control Systems with Performance Optimization, 2013.

[2] X. Zhao, L. Zhang, P. Shi, and H. Karimi, "Robust control of continuous-time systems with state-dependent uncertainties and its application to electronic circuits," IEEE Transactions on Industrial Electronics, vol. 61, no. 8, pp. 4161-4170, 2014.

[3] Z. Li, Z. Fei, and H. Gao, "Stability and stabilisation of Markovian jump systems with time-varying delay: an input-output approach," IET Control Theory \& Applications, vol. 6, no. 17, pp. 2601-2610, 2012.

[4] S. Yin, X. Yang, and H. R. Karimi, "Data-driven adaptive observer for fault diagnosis," Mathematical Problems in Engineering, vol. 2012, Article ID 832836, 21 pages, 2012.

[5] J. Qiu, G. Feng, and H. Gao, "Fuzzy-model-based piecewise $H_{\infty}$ static-output-feedback controller design for networked nonlinear systems," IEEE Transactions on Fuzzy Systems, vol. 18, no. 5, pp. 919-934, 2010.

[6] J. Qiu, G. Feng, and H. Gao, "Observer-based piecewise affine output feedback controller synthesis of continuous-time T-S Fuzzy affine dynamic systems using quantized measurements," IEEE Transactions on Fuzzy Systems, vol. 20, no. 6, pp. 10461062, 2012.

[7] J. Qiu, G. Feng, and H. Gao, "Asynchronous output-feedback control of networked nonlinear systems with multiple packet dropouts: T-S fuzzy affine model-based approach," IEEE Transactions on Fuzzy Systems, vol. 19, no. 6, pp. 1014-1030, 2011.

[8] B. Xiao, Q. L. Hu, and Y. M. Zhang, "Fault-tolerant attitude control for flexible spacecraft without angular velocity magnitude measurement," Journal of Guidance, Control, and Dynamics, vol. 34, no. 5, pp. 1556-1561, 2011.

[9] B. Xiao, Q. Hu, and Y. Zhang, "Adaptive sliding mode fault tolerant attitude tracking control for flexible spacecraft under actuator saturation," IEEE Transactions on Control Systems Technology, vol. 20, no. 6, pp. 1605-1612, 2012.

[10] S. Yin, G. Wang, and H. R. Karimi, "Data-driven design of robust fault detection system for wind turbines," Mechatronics, vol. 24, no. 4, pp. 298-306, 2014.

[11] S. Yin, S. X. Ding, A. H. A. Sari, and H. Hao, "Data-driven monitoring for stochastic systems and its application on batch process," International Journal of Systems Science, vol. 44, no. 7, pp. 1366-1376, 2013.

[12] S. Yin, S. X. Ding, A. Haghani, H. Hao, and P. Zhang, "A comparison study of basic data-driven fault diagnosis and process monitoring methods on the benchmark Tennessee Eastman process," Journal of Process Control, vol. 22, no. 9, pp. 1567-1581, 2012.

[13] J. Wang, H. Liang, Z. Sun, S. Zhang, and M. Liu, "Finitetime control for spacecraft formation with dual-number-based description," Journal of Guidance, Control, and Dynamics, vol. 35, no. 3, pp. 950-962, 2012.
[14] L. B. Hu and A. Zhang, "Robust finite-time control allocation in spacecraft attitude stabilization under actuator," Robust FiniteTime Control Allocation in Spacecraft Attitude Stabilization under Actuator, vol. 73, p. 21, 2013.

[15] E. D. Jin and Z. W. Sun, "Robust controllers design with finite time convergence for rigid spacecraft attitude tracking control," Aerospace Science and Technology, vol. 12, no. 4, pp. 324-330, 2008.

[16] S. Li, Z. Wang, and S. Fei, "Comments on the paper: robust controllers design with finite time convergence for rigid spacecraft attitude tracking control," Aerospace Science and Technology, vol. 15, no. 3, pp. 193-195, 2011.

[17] Y. Su, C. Zheng, and P. C. Müller, "Global continuous finitetime output feedback regulation of robot manipulators," in Proceeding of the IEEE International Conference on Robotics and Automation (ICRA '08), pp. 3383-3388, Pasadena, Calif, USA, May 2008.

[18] H. Du, S. Li, and C. Qian, "Finite-time attitude tracking control of spacecraft with application to attitude synchronization," IEEE Transactions on Automatic Control, vol. 56, no. 11, pp. 2711-2717, 2011.

[19] H. B. Du and S. H. Li, "Finite-time attitude stabilization for a spacecraft using homogeneous method," Journal of Guidance, Control, and Dynamics, vol. 35, no. 3, pp. 740-748, 2012.

[20] L. Rosier, "Homogeneous Lyapunov function for homogeneous continuous vector fields," Systems \& Control Letters, vol. 19, no. 6, pp. 467-473, 1992.

[21] S. Yu, X. Yu, B. Shirinzadeh, and Z. Man, "Continuous finitetime control for robotic manipulators with terminal sliding mode," Automatica, vol. 41, no. 11, pp. 1957-1964, 2005.

[22] X. H. Chen, F. Gao, and J. X. Qian, "Nonlinear adaptive control based on RBF networks and multi-model method," in Proceedings of the American Control Conference, vol. 3, pp. 15631567, Albuquerque, NM, USA, June 1997.

[23] D. M. Dawson, Nonlinear Control of Wheeled Mobile Robots, Springer, New York, NY, USA, 2001. 


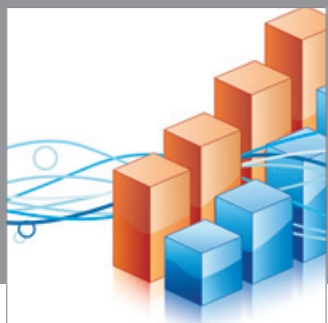

Advances in

Operations Research

mansans

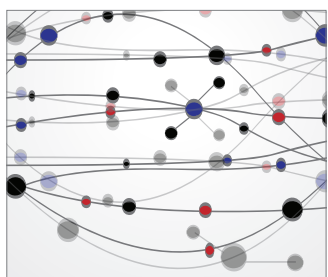

The Scientific World Journal
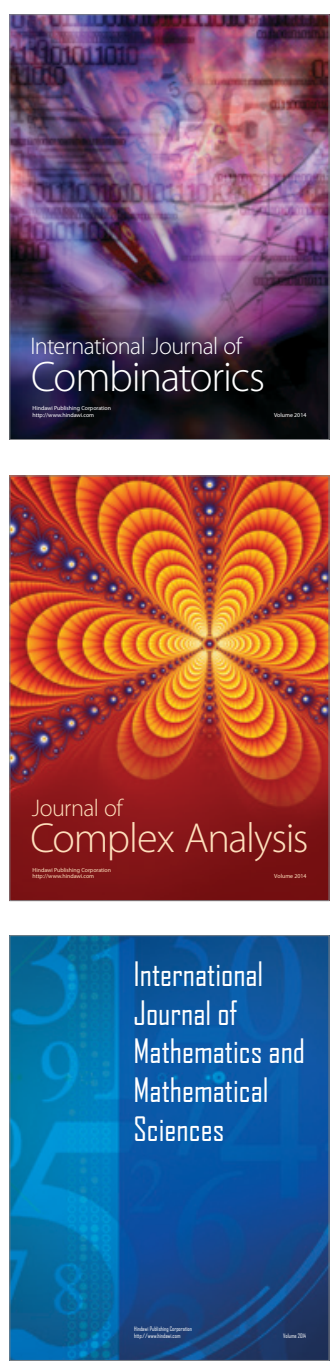
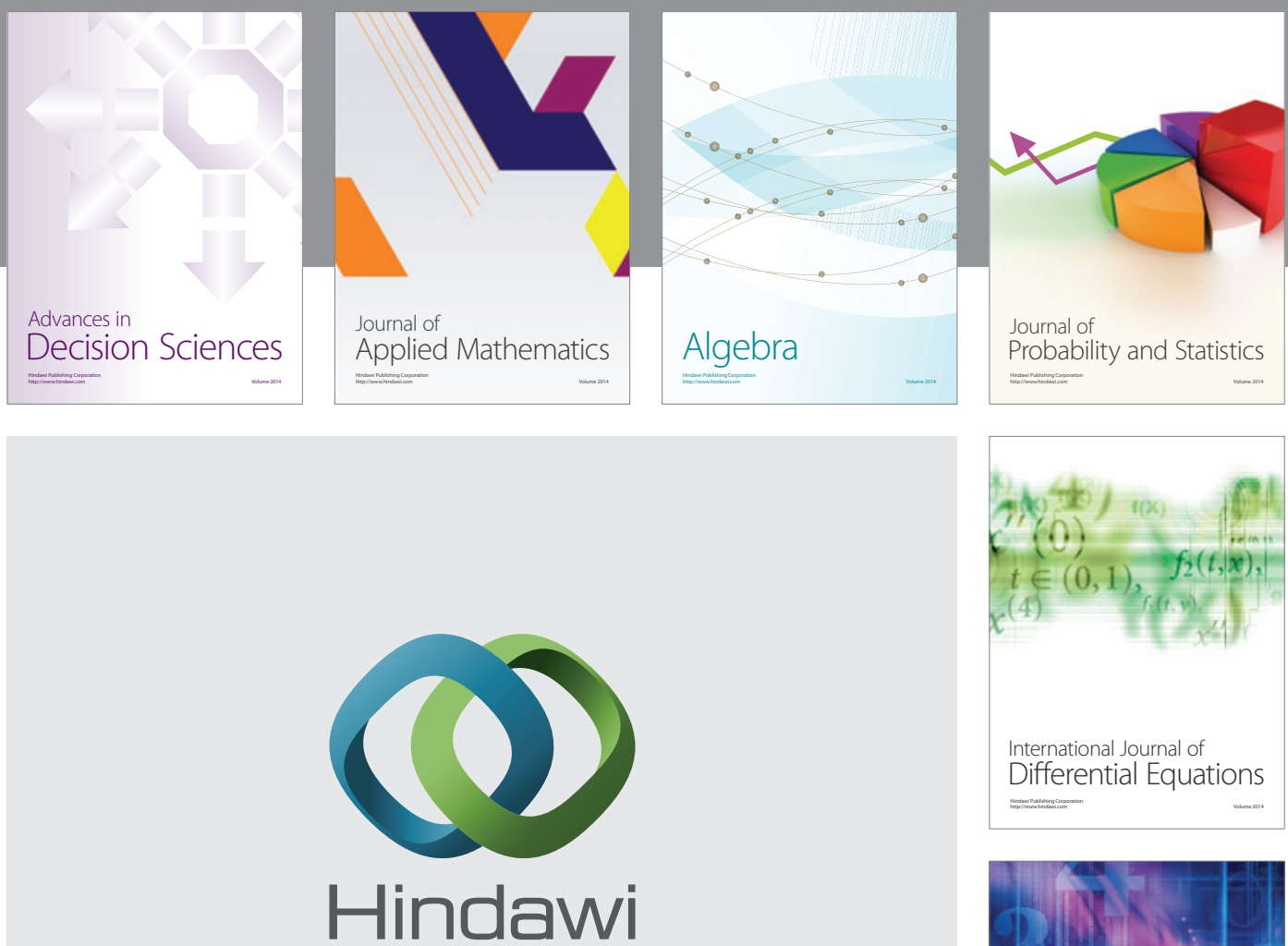

Submit your manuscripts at http://www.hindawi.com
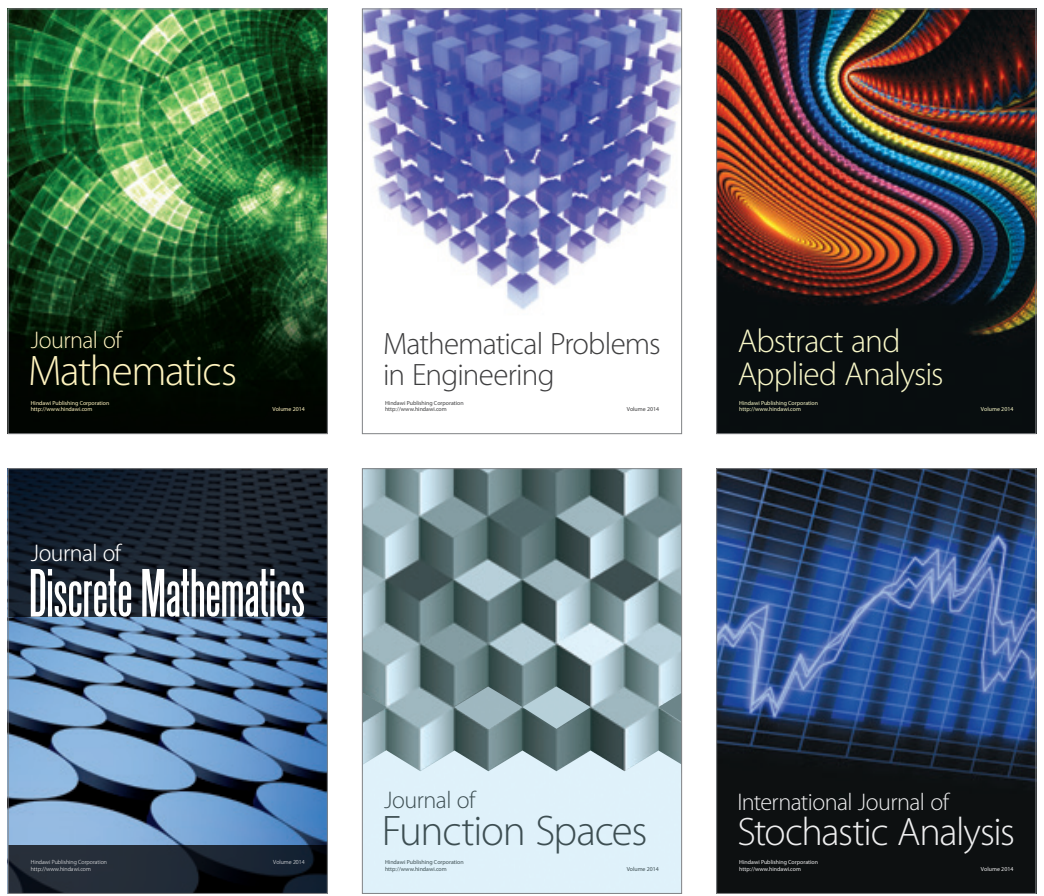

Journal of

Function Spaces

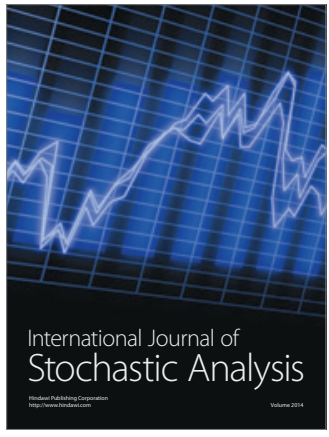

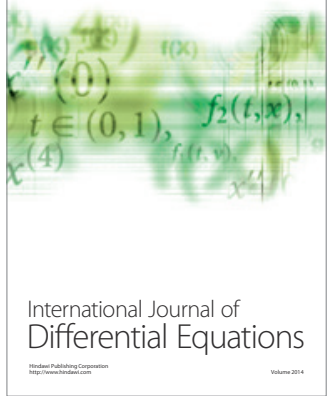
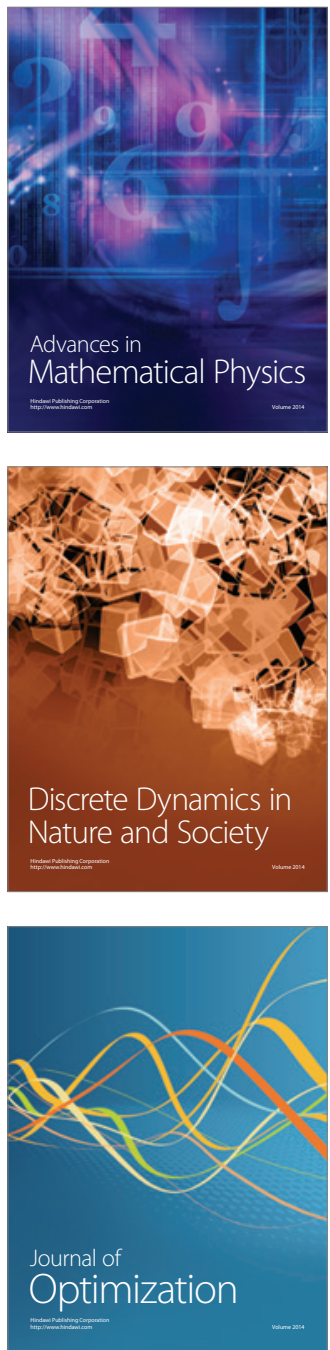